\title{
Automatic mineral identification using genetic programming
}

\author{
B.J. Ross ${ }^{1}$, F. Fueten ${ }^{2}$, D.Y. Yashkir ${ }^{1}$ \\ 1 Department of Computer Science, Brock University, St. Catharines, Ontario L2S 3A1, Canada; e-mail: bross@ cosc.brocku.ca \\ 2 Department of Earth Sciences, Brock University, St. Catharines, Ontario L2S 3A1, Canada
}

Received: 18 October 1999 / Accepted: 20 January 2001

\begin{abstract}
Automatic mineral identification using evolutionary computation technology is discussed. Thin sections of mineral samples are photographed digitally using a computer-controlled rotating polarizer stage on a petrographic microscope. A suite of image processing functions is applied to the images. Filtered image data for identified mineral grains is then selected for use as training data for a genetic programming system, which automatically synthesizes computer programs that identify these grains. The evolved programs use a decision-tree structure that compares the mineral image values with one other, resulting in a thresholding analysis of the multi-dimensional colour and textural space of the mineral images.
\end{abstract}

Key words: Mineral classification - Genetic programming - Feature space thresholding

\section{Introduction}

Most rocks are composed of microscopic-sized minerals that can be identified by a variety of methods. The most common method of manual mineral identification involves mounting a thin-section, 30- $\mu \mathrm{m}$-thick slice of the rock on a plate of glass, and viewing under a specialized microscope. At that sample thickness, light will pass through the rock and a geologist can examine the minerals under different lighting conditions, involving one or two polarizing filters. Competent mineral identification is a task that usually requires years of experience. An expert will use various visual cues; for example, colour, texture and the interaction of the crystal lattice with different directions of polarized light. A geologist experienced in microscopic mineral identification can identify grains fairly accurately. While a number of other techniques for mineral identification are available (e.g. microprobe and $\mathrm{x}$-ray diffraction), the manual use of thin sections is still in many cases the fastest and cheapest method available.

Given the challenges inherent in mineral identification, the use of computer-aided tools for automatic mineral identification is worth consideration. This paper illustrates how

Correspondence to: B.J. Ross computer vision and artificial intelligence technologies can be used to identify mineral grains automatically from digital images.

Automatic mineral identification is a multiple-stage process. Thin sections of mineral samples are photographed digitally using a computer-controlled rotating-polarizer petrographic microscope. These images are processed through a series of image processing filters, which results in a set of filtered images that encapsulate the original image features in more compact and convenient representations. One of these filtered images consists of grain edges, in which a graphical border surrounds each grain. When these grain boundaries are overlaid onto the other filtered images, it is immediately apparent that identifiable combinations of filtered image features characterize particular types of mineral specimens. These features are similar to those used by geologists when manually identifying a mineral in a thin section. Therefore, once the image features are obtained for the grains in a sample, that information should be sufficient for identifying the type of minerals comprising those grains.

Once the image data for a set of mineral images is extracted and filtered, it is put into a database of grain specimens. The problem then becomes one of characterizing minerals based on their data within this database. This is a classical problem for artificial intelligence, and a variety of artificial intelligence technologies are pertinent to its solution. First, it can be viewed as a classification problem, in which the features of a presented object are examined and used to assign the object to one of a predefined set of classes (Berry and Linoff 1997). Classification problems typically use well-defined descriptions of the classes, and a training set of examples of these classes. A set of grain data from the database can be used to derive descriptions of grain characteristics for use in deriving a model for mineral identification.

The problem can also be viewed as one in inductive concept learning, in which a generalization is derived from a finite set of examples (Luger and Stubblefield 1998). The task for inductive learning algorithms is to derive useful generalizations from the training examples. Over-generalization means that imprecise and inaccurate descriptions will result, while under-generalization will derive models that are too 
tightly bound to the training set's idiosyncrasies to be of general use for other types of mineral. Considerable machinelearning research has been undertaken in inductive learning, the most famous example being Quinlan's ID3 inductive learning algorithm (Quinlan 1986).

Another pertinent machine-learning paradigm is evolutionary computation (Holland 1992; Bäck 1996; Michalewicz 1996; Mitchell 1996; Bäck et al. 1997). Machine learning problems are characterizable as search problems, in which a possibly infinite space of model descriptions is searched in an attempt to find a description that best characterizes a set of training data. The evolutionary computation approach to machine learning uses a simulation of natural Darwinian evolution to search a space of model descriptions in the quest for one that satisfies the problem requirements.

This research solves the mineral identification problem using a specialized branch of evolutionary computation called genetic programming (GP) (Koza 1992). GP evolves a collection of data structures that can be interpreted as computer programs. As the search proceeds, increasingly more useful programs are built, until hopefully one evolves that solves the problem specification to a desired level of performance.

In this paper, GP is used to automatically synthesize computer programs that identify minerals from their grain features. The programs to be evolved take as input the filtered image data for a grain, examine and process that data, and respond with an identification of the mineral represented by that grain data. More precisely, each program synthesized is specialized for identifying one specific mineral that can appear in a thin-section sample. When one of these programs is supplied a set of data for a grain, it will determine if that grain corresponds to the mineral that the program is engineered to recognize. Therefore, all the grains in a thin section can be identified if the grain data is given to each identification program in succession.

An outline of the paper is as follows. Section 2 is a background discussion of the mineral identification problem and the rotating polarizer stage microscope. Section 3 discusses the image processing performed on the polarized images. The essentials of GP are reviewed in Sect. 4. The application of GP to mineral identification is discussed in Sect. 5. This includes a discussion of evolutionary strategies and the parameters used, and the results obtained. A critical discussion concludes the paper in Sect.6, which includes a comparison with other research employing neural networks (NNs) for mineral identification.

\section{Mineral identification}

The petrographic microscope is a commonly used tool for manual mineral identification in thin sections; however, its use for image processing has been limited (Fabbri 1984; Petruk 1989; Launeau et al. 1990; Pfleiderer et al. 1992; Starkey and Samantary 1993). Minerals are observed primarily under two different lighting conditions. In plane-polarized light, a single polarizing filter is placed below the thin section, while under cross-polarized illumination, one polarizer is placed above and one below the sample, with their polarizing directions at $90^{\circ}$ to each other. While a number of specialized tests can be performed on the sample, an experienced operator can often identify minerals by the nature of the change in appearance of the mineral as the sample is rotated with respect to the fixed polarizers. The use of optical automated mineral identification systems has been limited by several problems. In plane-polarized light, many minerals are colourless, which makes it impossible to distinguish grain boundaries between two adjacent colourless grains. Similarly, in cross-polarized light, the colour - referred to as interference colour - displayed depends on the mineral type, the orientation of the crystal lattice of the grain with respect to the polarizers and the thickness of the thin section. Hence, the appearance of a single grain may vary from black to brightly coloured, depending on the orientation of its lattice to the polarizers. This problem is overcome by rotating the microscope stage with respect to the fixed polarizers and observing the full range of colours that a grain undergoes. The human brain and vision system have no problem keeping track of individual grains as they rotate around the field of view. Unfortunately this procedure is a major obstacle for an image processing system, as the computer has to track the behaviour of a point within a grain in colour space, as well as the motion of that point as the thin section is rotated. Hence automated mineral identification systems (Launeau et al. 1994; Marschallinger 1997) are based on scanned images and use the natural colour of the mineral.

The rotating-polarizer microscope stage (Fueten 1997) was designed specifically as an addition to the standard petrographic microscope, to overcome some of its inherent problems. The stage works in conjunction with a video capture board and allows the thin section to remain fixed while the polarizers are rotated. This greatly enhances the usage of standard image processing techniques on thin sections for the segmentation, measurement and identification of the mineral.

\section{Image processing of polarized thin-section images}

Sampling of minerals is performed by repeatedly grabbing a frame, extracting data and rotating the polarizers. The normal sampling procedure rotates the polarizers from $0^{\circ}$ to $180^{\circ}$ in $0.9^{\circ}$ increments (200 steps) under both plane- and crosspolarized light. A composite data set is then constructed from this information. An example thin-section image is shown in Fig. 1, which has the maximum plane intensity for a specimen.

\subsection{Data collected using cross-polarized light}

The maximum intensity image contains the maximum intensity values as defined in hue, saturation and intensity (HSI) space. These correspond to the maximum interference colour that every pixel attained during a $180^{\circ}$ rotation of the polarizers. Intensity variations are related directly to the orientation of the crystal lattice with respect to the plane of the thin section. Variations in intensity due to the orientation of the polarizers, which are seen in single images, are eliminated by the sampling procedure. The maximum position image 


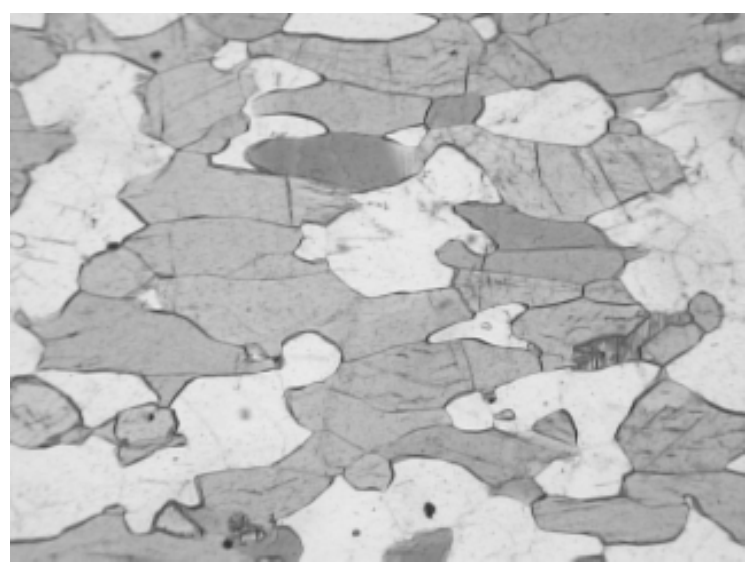

Fig. 1. Maximum plane intensity

is a greyscale image (with a $0-200$ intensity range) indicating that the polarizer position at which each pixel reached the maximum intensity. Position values record the orientation of the polarizing filters (i.e. the step number) when a pixel reaches its maximum value. The gradient image is a representation of the accumulated sum of the maximum difference in intensity between the horizontal and vertical directions. The image is then rescaled to a 0-255 range (for display purposes) and serves as the input of the segmentation routine as presented by Goodchild and Fueten (1998).

\subsection{Data collected using plane-polarized light}

A maximum intensity image is obtained under plane-polarized light. In addition, a minimum intensity image and a minimum position image are constructed similarly to the maximum intensity and position images under crosspolarized light. The advantage in having both maximum and minimum intensity images is that they can be used to obtain a measure of pleochroism, which is the variation in colour of a mineral during rotation under plane-polarized light.

\subsection{Parameter extraction}

It is relatively easy for the human brain to distinguish between different minerals by looking at qualitative features, but it is not obvious which numerical measurements can accomplish the same task. While their shape can be used to identify minerals, this is not generally the case. Hence it was decided that identification should restrict itself to visual parameters, such as colour and texture that can be extracted from each mineral. Mineral identification has to proceed on a grain-by-grain rather than a pixel-by-pixel basis, hence the images were segmented and the parameters were calculated on the basis of the pixels contained within a grain. A variety of colour and texture parameters were computed from the data set.

\subsubsection{Colour parameters}

Each image is captured using red, green and blue (RGB) components. However, experimentation determined the RGB colour model was very sensitive to fluctuations of the light source. Converting the RGB components to HSI space detaches the intensity component from the colour information and reduces the effects of variable lighting. The hue component provides a measurement that directly determines the colour of the grain, while the saturation yields the depth of that colour.

Pleochroism is a fundamental mineral characteristic that provides important information during manual mineral identification. Hence, a pleochroism parameter was defined as the difference between the modes of the intensity of the maximum intensity and the minimum intensity of the plane light images. For simplicity, any changes in the hue or saturation between the two plane-polarized-light intensity images were ignored.

\subsubsection{Texture parameters}

Colour is not a uniquely identifying characteristic of minerals. Several characteristics of minerals require special tests, which involve the insertion of a special lens or filter. However, an experienced petrologist can in many cases identify minerals using subtle colour-independent features such as undulatory extinction or small amounts of alteration without having to resort to specialized tests. Undulatory extinction, which results from a bend lattice and produces a series of dark bands crossing the crystal during rotation, would not manifest itself in colour images but would appear in position images and can be quantified by textural parameters. Autio et al. (1999) and Thompson et al. (2001) successfully used texture parameters to classify rock textures for a variety of purposes.

The intensity component of the colour images and the values of position images were used to calculate four standard texture parameters. Texture parameters are calculated using a co-occurrence matrix that is calculated for each grain. A co-occurrence matrix, representing a twodimensional histogram, defines a $P[i, j]$ value which corresponds to the number of pixels with the values $i$ and $j$ separated by the displacement vector $(1,1)$. This displacement expressed as an image mask is the $2 \times 2$ identity matrix. Using this normalized matrix $(P)$ the following measurements are calculated (Jain et al. 1995):

$$
\begin{array}{ll}
\text { Contrast } & =\Sigma \Sigma(\mathrm{i}-\mathrm{j})^{2} \mathrm{P}[\mathrm{i}, \mathrm{j}] \\
\text { Entropy } & =-\Sigma \Sigma \mathrm{P}[\mathrm{i}, \mathrm{j}] \log (\mathrm{P}[\mathrm{i}, \mathrm{j}]) \\
\text { Energy } & =\Sigma \Sigma \mathrm{P}^{2}[\mathrm{i}, \mathrm{j}] \\
\text { Homogeneity } & =\Sigma \Sigma \mathrm{P}[\mathrm{i}, \mathrm{j}] /(1+|\mathrm{i}-\mathrm{j}|)
\end{array}
$$

A total of 35 colour and texture parameters (Table 1) were extracted from the data set.

\section{Genetic programming}

Genetic programming (Koza 1992; Banzhaf et al. 1998; Whitley 2000) is an evolutionary computation paradigm in which programs are evolved using a genetic algorithm (GA). The basic GA is given in Fig. 2. GA and GP are characterized by the following: 
Table 1. Image parameters ( crossed, cross-polarized light; plane, planepolarized light)

$\begin{array}{lc}\text { Maximum crossed intensity: } & \text { Maximum crossed position: } \\ \text { p1. Hue } & \text { p22. Contrast } \\ \text { p2. Saturation } & \text { p23. Entropy } \\ \text { p3. Intensity } & \text { p24. Energy } \\ \text { p4. Contrast } & \text { p25. Homogeneity } \\ \text { p5. Entropy } & \\ \text { p6. Energy } & \text { Minimum plane position: } \\ \text { p7. Homogeneity } & \text { p26. Contrast } \\ \text { Maximum plane intensity: } & \text { p27. Entropy } \\ \text { p8. Hue } & \text { p28. Energy } \\ \text { p9. Saturation } & \text { p29. Homogeneity } \\ \text { p10. Intensity } & \\ \text { p11. Contrast } & \text { Gradient: } \\ \text { p12. Entropy } & \text { p30. Intensity } \\ \text { p13. Energy } & \text { p31. Contrast } \\ \text { p14. Homogeneity } & \text { p32. Entropy } \\ \text { Minimum plane intensity: } & \text { p33. Energy } \\ \text { p15. Hue } & \text { p34. Homogeneity } \\ \text { p16. Saturation } & \text { Pleochroism: } \\ \text { p17. Intensity } & \text { p35. Pleochroism } \\ \text { p18. Contrast } & \\ \text { p19. Entropy } & \\ \text { p20. Energy } & \\ \text { p21. Homogeneity } & \end{array}$

1. Initialize: Generate initial population.

2. Evolution:

Loop while current generation < maximum generations and fitness of best individual not considered a solution \{ Loop while new population size < maximum population size \{ - Select a genetic operation probabilistically: --> Crossover:

- Select two individuals based on fitness.

- Perform crossover.

- Evaluate the fitness of the offspring. --> Mutation:

- Add offspring to new population.

- Select one individual based on fitness.

- Perform mutation.

- Evaluate the fitness of the offspring.

- Add offspring to new population.

\}

- Increment generation counter.

3. Output: Print best solution obtained.

Fig. 2. Genetic algorithm

1. An initial population of random individuals, which in the case of GP are randomly constructed programs.

2. A finite number of generations, each of which results in a new or replenished population of individuals.

3. A problem-dependent fitness function, which takes an individual and gives it a numeric score indicative of that individual's ability to solve a problem at hand.
4. A fitness-proportional selection scheme, in which programs are selected for reproduction in proportion to their fitness.

5. Reproduction operations, usually the crossover and mutation operations, which take selected program(s) and generate offspring for the next generation.

The essential difference between GP and GA is the denotation of individuals in the population. A pure GA uses genotypes that are fixed-length bit strings, and which must be decoded into a phenotype for the problem being solved. A GP uses a variable-length computer program genotype, which is directly executable by some interpreter (typically LISP). The use of programming code as the genotype has been found to be a robust and powerful means of solving many problems (e.g. Whitley 2000).

The two main reproduction operators used in GP are crossover and mutation. Crossover permits the genetic combination of program code from programs into their offspring, and hence acts as the means for inheritance of desirable traits during evolution. Crossover takes two selected programs, finds a random crossover point in each program's internal representation (normally a parse tree), and swaps the subtrees at those crossover points. The crossover points must be selected so that the resulting offspring are syntactically correct. With the program structure in Koza (1992), this means that if a subtree with a non-terminal root (function label) is selected in one program, it is swapped with a subtree with a non-terminal root from the other program.

To perform mutation, a random mutation point is found in a selected program, and the subtree at that node is replaced with a new, randomly generated tree. This is the means by which new genetic traits can be introduced into the population during evolution. Although crossover and mutation preserve the grammatical integrity of programs, the user must ensure closure that the resulting programs are always executable. So long as closure is maintained, all programs derivable by the GP system will be executable by the fitness function, and hence their fitness will be ascertainable.

\section{Evolution of mineral identification programs}

\subsection{Data preparation}

As discussed in Sect. 3, the raw polarized images from the rotating-polarizer stage microscope are processed through a number of filters, resulting in a set of 35 distinct data parameters. These parameters are computed for grain within the images. In order to extract the training data, a geologist manually inspected and identified a series of minerals from a set of thin sections. The data vectors of different minerals and their identification are then saved in a grain database. This information is used by the GP-fitness evaluation procedure.

The grain data were taken from a set of 44 petrographic thin sections. Some of the sparser minerals on the thin sections could not be used, since there were not enough examples to process by the GP system. The minerals species and number of examples of each mineral examined here were: quartz (575), K-spar (369), plag (358), biotite (74), muscovite (36), hornblende (86), CPX (81), olivine (685), garnet (311), calcite (520) and opaque (189). The number 


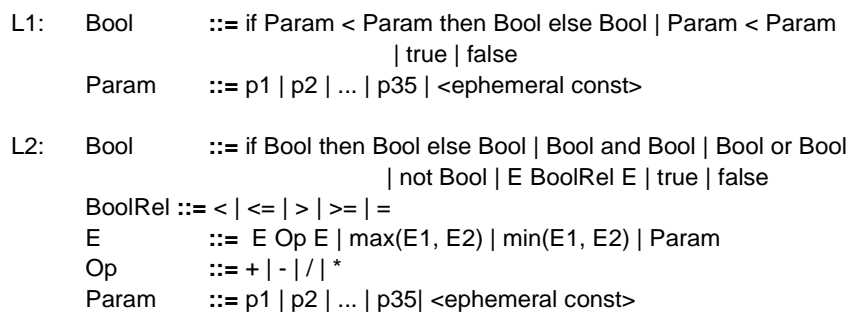

Fig. 3. Grammar of the GP languages

of example grains varied in these minerals. This affects the ability to run the GP system on them for larger sets of training examples.

\subsection{Target languages}

An important preparation step for GP is to identify a suitable target language in which to evolve programs. The success of evolution is dependent upon the use of a language that can adequately represent a solution to the problem being solved; should an inadequate language be used, there will be no means for the GP system to evolve a solution. In addition, higher-level languages can sometimes enhance evolution efficiency, since higher-level features can promote faster evolution of better quality programs. This must be balanced with efficiency of execution, however, since highlevel functions may produce a computational overhead that will slow the GP system considerably.

Two different languages were used for evolving mineral identification programs. The Backus-Naur grammar for the languages is in Fig. 3. This grammar is translated into a LISP-like notation for use by the GP system. The first language, L1, is a nested decision tree language, consisting of nestable If-Then statements, a relational operator " $<$ " over the 34 parameter values and the logical constants True and False. Both conditional If and relational " $<$ " statements are used since the latter permits decision trees to be leaner in size than if only nested "If"s were allowed. As can be seen in the grammar, an L1 program will performing a relational test on the parameter data, and will return a logical value as a result. The intention is for a mineral identification program written in L1 to return True if the grain parameters being analyzed conform to the particular mineral that that program is designed to identify, and False otherwise.

The second language, L2, is more complex. It is a superset of L1, introducing mathematical expressions over parameter values. Most of L2 is self-explanatory. The Min and Max operators return the minimal and maximal value of their pairs of arguments. All equality tests within relational expressions work with a degree of error within $10 \%$ of the left-hand parameter. For example, in the expression " $a=b$ ", if $b$ is within $10 \%$ of $a$ 's value, the expression returns True. All the operators must observe closure, by executing with any possible argument value. The only operator this affects is the "/" division operator, which must handle zero-value denominators. It is coded to return " 0 " in these instances. Ephemeral constants are floating-point numbers between 0.0 and 1.0, which are initially generated with a random num- ber generator, but thereafter retain their initialized values throughout their existence.

\subsection{Fitness strategy}

Each program to be evolved is to identify one of the mineral types resident on the thin-section images. Let this mineral be designated as mineral $\mathrm{M}$. Once a mineral is selected as a goal for evolution, the grain database is segregated into two sets of data: training and testing. Training data are the grain feature vectors (henceforth called "grains"), which will be used during GP evolution by the fitness evaluator. Let $N$ be the total number of minerals. The training set for a mineral consists of a random selection of $K$ grains corresponding to the mineral being identified, plus a random selection of $K$ grains for each of the other minerals. This yields a total of $N \times K$ grains to be used as training examples.

The fitness function used during evolution is the following:

$\frac{1}{2}\left(\frac{\# \text { hits correct }}{K}+\frac{\# \text { misses correct }}{(N-1) K}\right)$

A hit is when the program-identifying mineral $\mathrm{M}$ identifies a grain as belonging to $\mathrm{M}$, or in other words, the program returns True. Similarly, a miss is when the program returns False. This formula therefore computes the percentage of correctly identified grains of mineral $\mathrm{M}$, and the percentage of grains correctly identified as not being mineral $\mathrm{M}$. The sum of these terms is scaled by $1 / 2$, to keep the range of values between 0 and 1 . This implies that a value of 1 means that a program has perfectly identified all the grains in the training set, while 0 implies that no grains were correctly identified. Note that the equation is weighted towards awarding correct hits. This is because there are far fewer positive examples than negative examples in the training set. Otherwise, if all the grains were evaluated with the same weight, a program that returned False for all grains would have a relatively high score of $(N-1) / N$ percent, which negatively affects evolution.

The testing set is used to measure the performance of programs on new data not seen during training. The testing set is a good way to ensure that a program has not been over-trained to recognize only its training data, and also to verify that the training data is representative of the problem at hand. The testing set is defined to be the rest of the grain database not used for training. The testing formula is similar to the fitness formula:

$\frac{1}{2}\left(\frac{\# \text { hits correct }}{P}+\frac{\# \text { misses correct }}{Q}\right)$

where $P$ is the total number of grains for mineral $\mathrm{M}$ in the testing set, and $Q$ is the total number of other grains. Again, this formula is weighted towards favouring correct hits, because there may be fewer grains for $M$ than the rest of the minerals. Also note that the testing set does not necessarily have a balanced number of grains for different minerals, since it is defined to be whatever is left in the database after the training set has been extracted. Since some mineral specimens are far rarer than others, the testing measurements for various minerals should not be directly compared with one 
Table 2. GP parameters

\begin{tabular}{ll}
\hline Parameter & Value \\
\hline Terminals, non-terminals & (see Fig. 3) \\
Fitness function & Number of correct identifications \\
Training set size & (i) $34+, 340-$ (ii) $70+, 420-$ \\
Generation type & Generational \\
Selection scheme & Tournament, size = 7 \\
Population size & 500 \\
Maximum generations & 50 \\
Runs per experiment & 20 \\
Initial population tree size & Ramped half and half, $2 \leq$ size $\leq$ \\
Maximum tree size & 17 \\
Probability crossover & 0.95 \\
Probability mutation & 0.05 \\
Probability internal & \\
(external) crossover & $0.1(0.9)$ \\
\hline
\end{tabular}

another. The important relationship to note is how well the testing and training values correlate with each another.

\subsection{Other experimental details}

Table 2 lists other parameters for the GP experiments. Most of these parameters are well known from the GP literature, and will not be discussed further. The terminals and nonterminals depend upon whether language L1 or L2 is used (see Fig. 3).

Two sets of experiments were undertaken. The first set of runs evolved identification programs for all the minerals, and used a minimal training set of 34 positive examples and 340 negative examples per run. A set of training data for a single run was extracted pseudo-randomly from the database. The second set of runs doubled the number of positive examples to 70 . In doing this, four of the eleven minerals (biotite, muscovite, hornblende and CPX) had to be excluded, because they provided insufficient numbers of positive examples in the grain database to permit accurate training and testing.

The GP system used is the lil-gp 1.1 system (Zongker and Punch 1995). The version used is a patched one by Sean Luke, which adds strong typing. Typing permits a more constrained search during evolution, as well as less stringent requirements for closure for languages L1 and L2 (Montana 1995). The system is compiled with GNU C on the Silicon Graphics IRIX 6.3 operating system. This C implementation of GP was chosen for its efficiency when processing the large example sets in this application.

\subsection{Results}

Table 3 shows the final results of the GP runs. For comparison, the results of testing performance values for the same data by an NN (Thompson et al. 2001) are given in the last column.

The more complex language L2, with its additional floating-point arithmetic, did not lend any great advantages to the quality of solutions evolved in the simpler decision language L1. Although 7 of the best mineral identifiers in

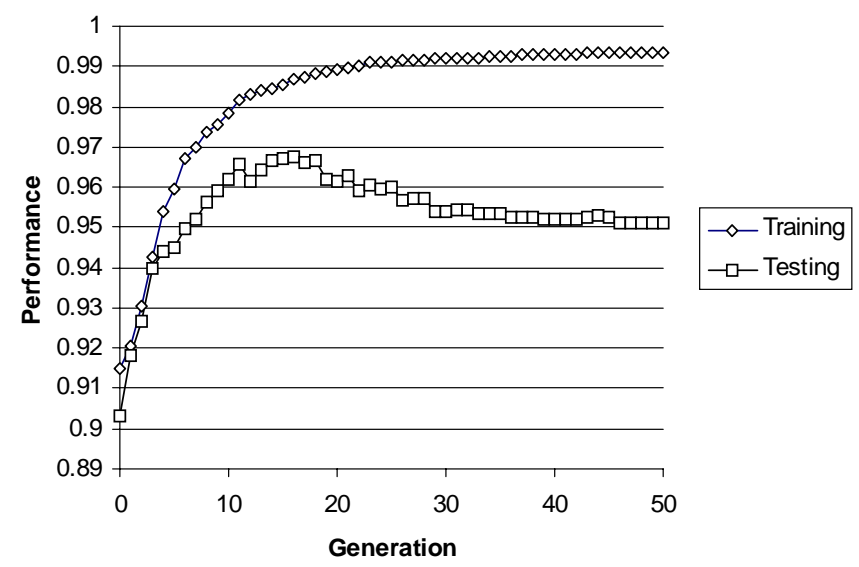

Fig. 4. Opaque evolution L2, $K=34$, av. 20 runs

the table are written in L2, their scores are not outstandingly better than the equivalent L1 solutions. L2 was in fact more expensive to use during the GP runs, as floating-point arithmetic is computationally more expensive to execute. Hence L2 runs took between two to three times longer to execute than $\mathrm{L} 1$ runs.

Using more positive grain examples was normally advantageous to the average quality of solutions obtained. However, in most of the cases for L1, the solutions obtained for $K=34$ were marginally better than those for $K=70$. This is probably a combination of the particular samples of grain used in these cases, being more reflective of the entire grain set as a whole, as well as inherent statistical margins of error. This phenomenon did not occur with L2. In any case, the differences in errors between the values for $K=34$ and $K=70$ are not exceptional, which implies that the examples selected for $K=34$ were representative of the database as a whole.

Another factor affecting the overall performance is that the grain database itself had errors. A geologist manually identified the thousands of grains in this database, and some grains may have been misidentified. When such an erroneous grain happens to be selected for training, it can introduce noise into the evolution process. This is especially acute when such a misidentified grain is blatantly different in visual characteristics to the rest of the mineral to which that grain belongs. Obviously, this problem is more pronounced when smaller training sets are used.

One phenomenon observed was that the runs using $K=34$ often suffered from over-training effects. In Fig. 4 it can be seen that the testing fitness declines after approximately generation 15 , while the training fitness continues to improve. This implies that the population is not correctly generalizing from the training set. Over-training did not occur for the runs with $K=70$.

A variety of different styles of programs were evolved. One example L2 program $(K=70)$ that identified garnet with a testing performance of $97.6 \%$ is the following simple program:

$\mathrm{p} 3<(\mathrm{p} 16-\mathrm{p} 21) * \mathrm{p} 16$

This program has 7 nodes and a depth of 3 (see Table 1 for the parameters denoted in the expression). This program is unusually concise compared to others in the runs for this 
Table 3. Results (values are percentages; $N N$, neural network)

\begin{tabular}{|c|c|c|c|c|c|c|c|c|c|c|}
\hline \multirow[b]{3}{*}{ Mineral } & & \multicolumn{4}{|c|}{ L1 } & \multicolumn{4}{|c|}{ L2 } & \multirow{3}{*}{$\begin{array}{l}\text { NN } \\
K=74 \\
\text { Test }\end{array}$} \\
\hline & & \multicolumn{2}{|c|}{$K=34$} & \multicolumn{2}{|c|}{$K=70$} & \multicolumn{2}{|c|}{$K=34$} & \multicolumn{2}{|c|}{$K=70$} & \\
\hline & & Test & Train & Test & Train & Test & Train & Test & Train & \\
\hline \multirow{2}{*}{ Quartz } & Best: & 90.2 & 94.6 & 92.2 & 93.8 & 93.5 & 97.5 & 97.8 & 96.1 & 96.5 \\
\hline & Av.: & 84.7 & 90.9 & 85.7 & 88.9 & 88.3 & 93.7 & 90.1 & 92.5 & \\
\hline \multirow[t]{2}{*}{ K-spar } & Best: & 94.2 & 97.8 & 93.9 & 96.0 & 93.8 & 99.0 & 93.4 & 96.9 & 88.6 \\
\hline & Av.: & 89.7 & 95.9 & 91.0 & 93.5 & 90.7 & 97.8 & 90.8 & 93.9 & \\
\hline \multirow[t]{2}{*}{ Plag } & Best: & 86.2 & 91.8 & 84.7 & 93.8 & 83.3 & 95.7 & 85.4 & 94.3 & 89.1 \\
\hline & Av.: & 75.9 & 88.0 & 78.9 & 88.9 & 78.6 & 91.8 & 80.2 & 91.0 & \\
\hline \multirow[t]{2}{*}{ Biotite } & Best: & 98.1 & 98.1 & - & - & 96.6 & 99.4 & - & - & 97.3 \\
\hline & Av.: & 93.7 & 96.4 & & & 91.7 & 98.0 & & & \\
\hline \multirow[t]{2}{*}{ Muscovite } & Best: & 98.3 & 96.6 & - & - & 97.6 & 99.9 & - & - & - \\
\hline & Av.: & 74.8 & 94.1 & & & 78.0 & 97.9 & & & \\
\hline \multirow[t]{2}{*}{ Hornblende } & Best: & 91.4 & 97.7 & - & - & 92.8 & 98.5 & - & - & 95.4 \\
\hline & Av.: & 86.8 & 94.7 & & & 86.1 & 95.8 & & & \\
\hline \multirow[t]{2}{*}{ CPX } & Best: & 90.3 & 96.5 & - & - & 93.8 & 98.5 & - & - & 97.5 \\
\hline & Av.: & 81.0 & 88.1 & & & 84.9 & 92.8 & & & \\
\hline \multirow[t]{2}{*}{ Olivine } & Best: & 90.0 & 94.4 & 89.9 & 93.8 & 91.4 & 96.6 & 93.2 & 98.1 & 92.8 \\
\hline & Av.: & 83.0 & 88.6 & 86.6 & 90.0 & 84.4 & 92.6 & 89.4 & 95.1 & \\
\hline \multirow[t]{2}{*}{ Garnet } & Best: & 98.2 & 99.7 & 98.9 & 99.6 & 97.8 & 100 & 99.4 & 99.8 & 97.4 \\
\hline & Av.: & 96.0 & 98.3 & 97.6 & 98.1 & 95.8 & 98.9 & 97.1 & 98.6 & \\
\hline \multirow[t]{2}{*}{ Calcite } & Best: & 92.1 & 97.4 & 91.8 & 95.2 & 92.8 & 99.0 & 95.1 & 98.2 & 96.0 \\
\hline & Av.: & 84.4 & 93.6 & 88.5 & 93.0 & 86.2 & 96.3 & 91.2 & 95.9 & \\
\hline \multirow[t]{2}{*}{ Opaque } & Best: & 97.7 & 99.9 & 97.7 & 99.9 & 98.3 & 100 & 98.1 & 100 & 95.2 \\
\hline & Av.: & 93.6 & 98.9 & 96.6 & 99.4 & 95.2 & 99.3 & 96.5 & 99.7 & \\
\hline
\end{tabular}

set of experiments, which normally had tree sizes averaging 222 nodes (the largest tree had 500 nodes). For example, another program in this set of runs with 93 nodes and a testing performance of $98.0 \%$ is:

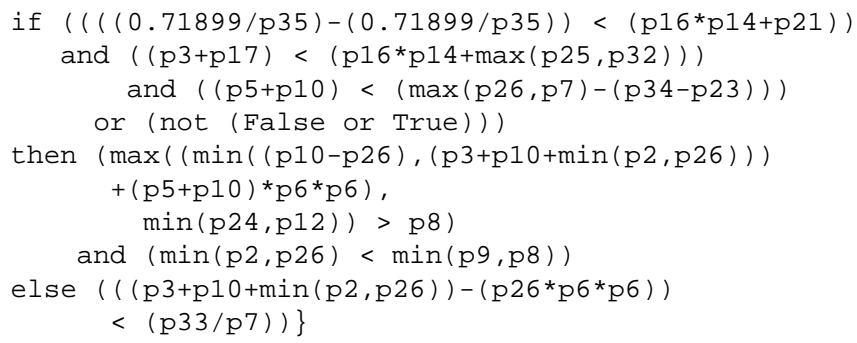

As is often to be expected with GP results, it is difficult to intuit the logic used in this program. However, human comprehension of a program is not a necessary condition for judging a program's merit or quality. Also note that there is some intron material ("junk DNA") that can be simplified. For example, the expression "(not (or False True))" simplifies to "False", which in turn can be used to simplify its parent expression.

Intron material is often prevalent in some solutions, which is evident when a superfluous number of unnecessary tests and terms exist. Consider the 57-node L1 solution, shown at the top of the next page, for identifying K-spar ( $K=34$, testing fitness $90.1 \%$ ). Removing redundant tests and simplifying expressions can remove 34 nodes from this solution:

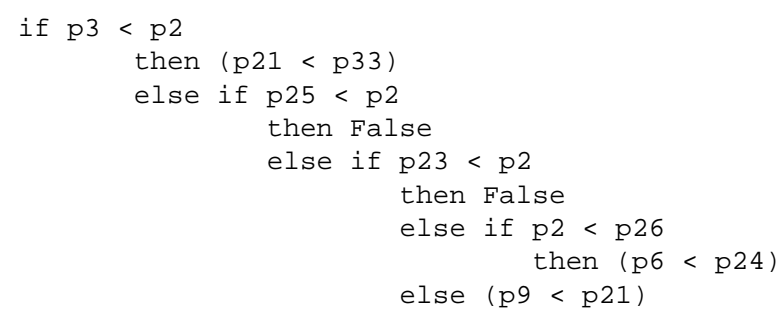

It is possible to implement an automatic simplifier that does the above transformation.

\section{Discussion}

The research reported in this paper successfully used GP to evolve mineral identification programs for use with images obtained with the rotating-polarizer microscope. The overall performance of the best mineral identifiers ranged from $86 \%$ to $98 \%$. These programs will be incorporated into an interactive workstation environment, which will automatically identify grains as selected by the user.

The GP performance is comparable to that obtained with a NN for mineral identification (Thompson et al. 2001). A direct comparison of these two different approaches is difficult to do, given the differences inherent between NNs 


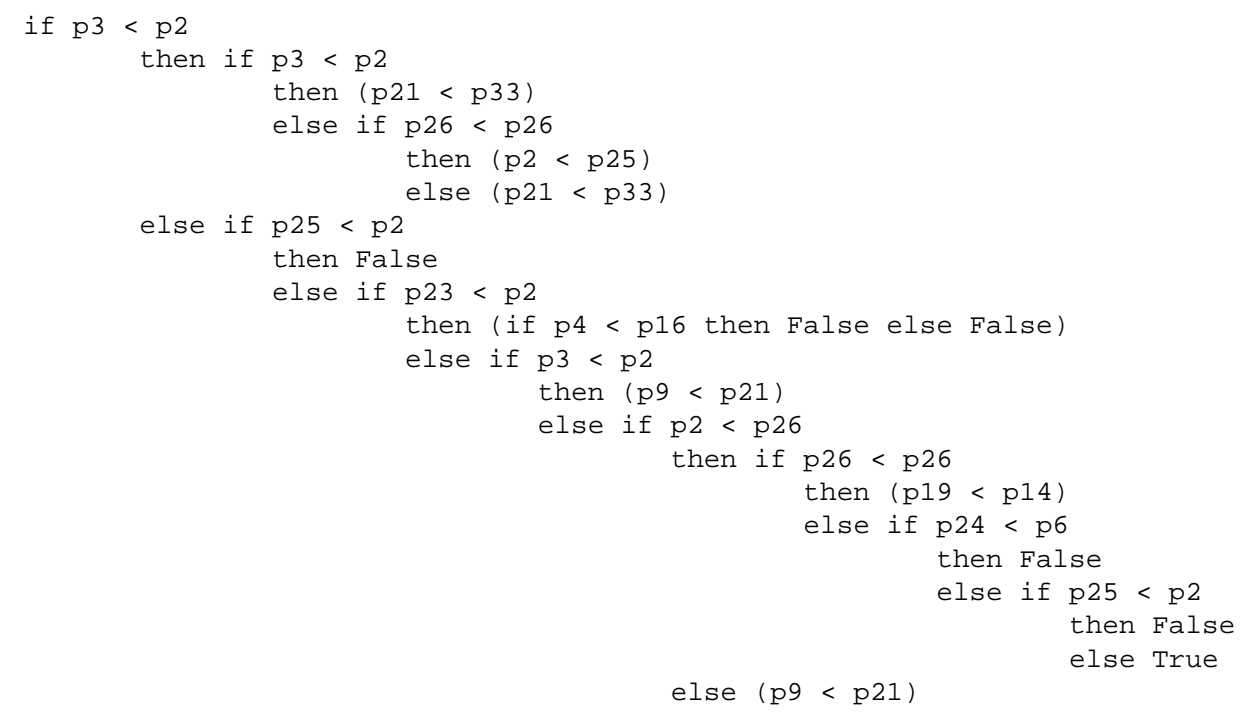

and genetic programs. The GP identifiers are specialized for each mineral, and hence are evolved separately. On the other hand, the $\mathrm{NN}$ is trained to identify all the minerals with one single network. The NN takes the 35 image parameters as input, as well as a code that identifies the mineral specimen for that grain. It also has 16 outputs, with one output per mineral specimen. The weights generated on these outputs identify the type of mineral corresponding to the input pattern. Hence the neural network is a "universal identifier" for the entire set of minerals.

To determine whether a universal mineral identifier could be evolved with GP, another GP experiment was undertaken. Rather than generate a logical True or False if a grain parameter matches a particular mineral, as was done earlier, these new programs would generate a label corresponding to the mineral matching the parameter data. A set of terminals corresponding to the set of mineral names was given to the system, and a training strategy similar to that used before was tried. These runs were unsuccessful in producing good quality identifiers, and the best solution obtained had a testing performance of $56.4 \%$ (training $=46.5 \%$ ). This is still better than the performance expected from a random identifier (testing $=9 \%$ ), but it is not nearly as accurate as the dedicated mineral identifiers. Part of the problem with this "universal identifier" approach is that it is difficult for evolution to ensure that all the mineral labels themselves are retained in the single program. During many runs, many mineral labels were lost throughout the population, which automatically prohibited the identification of those minerals by the programs in later generations. When this is added to the basic problem of evolving a useful identification strategy, the search becomes too challenging for GP. Hence the strategy undertaken in this paper of evolving individual mineral identifiers is the most sensible one for GP.

This is the first attempt at using evolutionary computation to identify minerals. The use of an NN for mineral identification has been described previously (Thompson et al. 2001). There are many examples of work elsewhere applying GP to pattern classification (Abramson and Hunter
1996; Gray et al. 1996; Ryu and Eick 1996; Zhao et al. 1996) and inductive learning (Siegel 1994; Freitas 1997).

Acknowledgements. This research was supported by NSERC operating grants 138467 and 0046210, and an NSERC undergraduate research grant.

\section{References}

Abramson M, Hunter L (1996) Classification using cultural co-evolution and genetic programming. In: Koza JR, Goldberg D, Fogel D, Riolo R (eds) Genetic Programming 1996: Proceedings of the First Annual Conference. MIT Press, Cambridge, Mass., pp 249-254

Autio J, Rantanen L, Visa A, Lukkarinen S (1999) The classification and characterisation of rock using texture analysis by co-occurrence matrices and the Hough transform. In: Proceedings Geovision 99: International Symposium on Imaging Applications in Geology, University of Liege, Belgium, May 6-7, 1999 pp 5-8

Bäck T (1996) Evolutionary algorithms in theory and practice. Oxford University Press, New York

Bäck T, Hammel U, Schwefel H-P (1997) Evolutionary Computation: comments on the History and Current State. IEEE Trans Evol Comput $1: 3-17$

Banzhaf W, Nordin P, Keller RE, Francone FD (1998) Genetic programming - an introduction. Kaufmann, San Francisco

Berry MJA, Linoff G (1997) Data mining techniques. Wiley, New York

Fabbri AG (1984) Image processing of geological data. Van NostrandReinhold, Wokingham, U.K.

Freitas AA (1997) A genetic programming framework for two data mining tasks: classification and generalized rule induction. In: Koza JR, Deb K, Dorigo M, Fogel D, Garzon M, Iba H, Riolo R (eds) Genetic Programming 1997: Proceedings of the Second Annual Conference. Kaufmann, San Francisco, pp 96-101

Fueten F (1997) A computer controlled rotating polarizer stage for the petrographic microscope. Comput Geosci 23:203-208

Goodchild JS, Fueten F (1998) Edge detection in petrographic images using the rotating polarizer stage. Comput Geosci 24:745-751

Gray HF, Maxwell RJ, Martinez-Perez I, Arus C, Cerdan S (1996) Genetic programming for classification of brain tumours from nuclear magnetic resonance biopsy spectra. In: Koza JR, Goldberg D, Fogel D, Riolo R (eds) Genetic Programming 1996: Proceedings of the First Annual Conference. MIT Press, Cambridge, Mass., p 424

Holland JH (1992) Adaptation in natural and artificial systems. MIT Press, Cambridge, Mass. 
Jain R, Kasturi R, Schunk BG (1995) Machine vision. McGraw-Hill, New York

Koza JR (1992) Genetic programming - on the programming of computers by means of natural selection. MIT Press, Cambridge, Mass.

Launeau P, Bouchez J-L, Benn K (1990) Shape preferred orientation of object populations: automatic analysis of digitized images. Tectonophysics 180:201-211

Launeau P, Cruden CA, Bouchez J-L (1994) Mineral recognition in digital images of rocks: a new approach using multichannel classification. Can Mineral 32: 919-933

Luger GF, Stubblefield WA (1998) Artificial intelligence - structures and strategies for complex problem solving. Addison-Wesley, Reading, Mass.

Marschallinger R (1997) Automatic mineral classification in the macroscopic scale. Comput Geosci 23:119-126

Michalewicz Z (1996) Genetic algorithms + data structures = evolution programs, 3rd edn. Springer, Berlin Heidelberg New York

Mitchell M (1996) An introduction to genetic algorithms. MIT Press, Cambridge, Mass.

Montana DJ (1995) Strongly typed genetic programming. Evol Comput 3:199-230

Petruk W (1989) Short course on image analysis applied to mineral and earth sciences. (Short course handbook, vol 16) Mineralogical Association of Canada, Ottawa

Pfleiderer S, Ball DGA, Bailey RC (1992) AUTO: A computer program for the determination of the two-dimensional auto-correlation function of digital images. Comput Geosci 19:825-829

Quinlan JR(1986) Induction of decision trees. Mach Learn 1:81-106

Ryu T-W, Eick CF (1996) MASSON: discovering commonalities in collection of objects using genetic programming. In: Koza JR, Goldberg D, Fogel D, Riolo R (eds) Genetic Programming 1996: Proceedings of the First Annual Conference. MIT Press, Cambridge, Mass., pp 200-208

Siegel EV (1994) Competitively evolving decision trees against fixed training cases for natural language processing. In: Kinnear KE Jr (ed) advances in genetic programming. MIT Press, Cambridge, Mass.

Starkey J, Samantary AK (1993) Edge detection in petrographic images. J Microsc 172:263-266

Thompson S, Fueten F, Bockus D (2001) Mineral identification using artificial neural networks and the rotating polarizer stage. Comput Geosci, Elsevier, in press

Whitley D, Goldberg D, Cantú-Paz E, Spector L, Parmee I, Beyer H-G (eds) (2000) Proceedings of the genetic and evolutionary computation conference. Kaufmann, San Francisco

Zhao J, Kearney G, Soper A (1996) Emotional expression classification by genetic programming. In: Late Breaking Papers at the Genetic Programming 1996 Conference. July 28-31, Stanford University, Stanford, CA, pp 197-202

Zongker D, Punch B (1995) LIL-GP 1.0 user's manual. Department of Computer Science, Michigan State University, East Lansing, MI

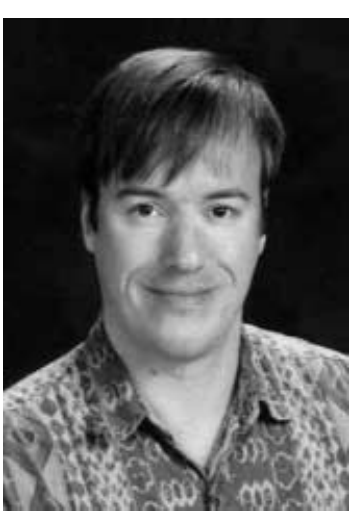

Brian Ross is an associate professor of computer science at Brock University, where he has worked since 1992. He obtained his BCSc at the University of Manitoba, Canada, in 1984, his MSc at the University of British Columbia, Canada, in 1988, and his $\mathrm{PhD}$ at the University of Edinburgh, Scotland, in 1992. His research interests include evolutionary computation, machine learning, language induction, concurrency and logic programming.

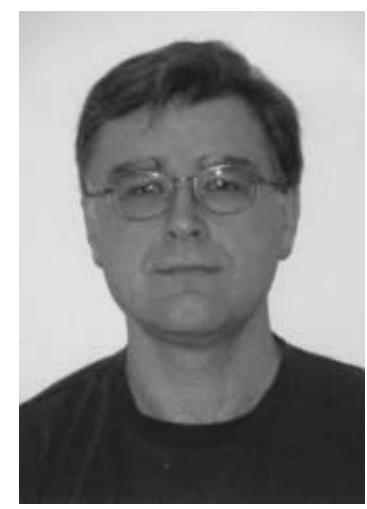

Frank Fueten received a BSc and MSc from the Department of Geology at McMaster University in Canada in 1982 and 1985 , respectively. Since graduating in 1990 with a PhD from the Department of Geology at the University of Toronto, he has been employed at Brock University, where he is an associate professor. His current research interests include the development of petrographic image processing techniques using the rotating polarizer stage.

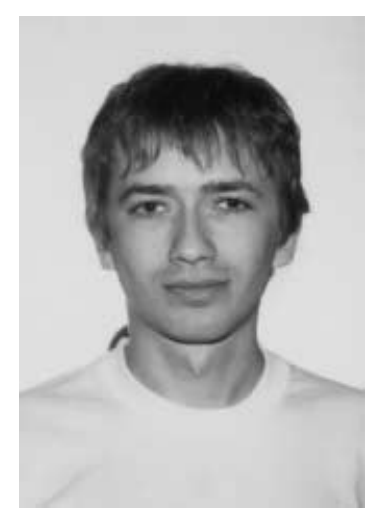

Dmytro Yashkir is a 4th year BSc Honours student in computer science at Brock University. His interests include evolutionary computation, machine learning and artificial intelligence applications in economics. 